\title{
Testing the companion hypothesis for the origin of the X-ray emission from intermediate-mass main-sequence stars ${ }^{\star}$
}

\author{
B. Stelzer ${ }^{1}$, N. Huélamo ${ }^{2}$, G. Micela ${ }^{1}$, and S. Hubrig ${ }^{2}$ \\ 1 INAF - Osservatorio Astronomico di Palermo, Piazza del Parlamento 1, 90134 Palermo, Italy \\ e-mail: stelzer@astropa.unipa.it \\ 2 European Southern Observatory, Casilla 19001, Santiago 19, Chile
}

Received 24 October 2005 / Accepted 20 February 2006

ABSTRACT

\begin{abstract}
Context. The X-ray emission from B-type main-sequence stars is a longstanding mystery in stellar coronal research. Since there is no theory at hand that explains intrinsic X-ray emission from intermediate-mass main-sequence stars, the observations have often been interpreted in terms of (unknown) late-type magnetically active companion stars.

Aims. Resolving the hypothesized companions requires high spatial resolution observations in the infrared and in X-rays. We use Chandra imaging observations to spatially resolve a sample of main-sequence B-type stars with recently discovered companions at arcsecond separation.

Methods. Our strategy is to search for X-ray emission at the position of both the B-type primary and the faint companion.

Results. We find that all spatially resolved companions are X-ray emitters, but seven out of eleven intermediate-mass stars are also $\mathrm{X}$-ray sources. If this emission is interpreted in terms of additional sub-arcsecond or spectroscopic companions, this implies a high multiplicity of B-type stars. Firm results on B star multiplicity pending, the alternative, that B stars produce intrinsic X-rays, cannot be discarded. An appropriate scenario would be a magnetically confined wind, as suggested for the X-ray emission of the magnetic Ap star IQ Aur. However, the only Ap star in the Chandra sample is not detected in X-rays, and therefore does not support this picture.
\end{abstract}

Key words. X-rays: stars - stars: early-type - stars: coronae - stars: activity

\section{Introduction}

After their discovery with the Einstein satellite (Cash \& Snow 1982; Caillault \& Zoonematkermani 1989) X-ray detections of A- and B-type stars repeatedly have been reported in the literature (see references in Stelzer et al. 2003), but their origin has remained a mystery.

Intermediate-mass stars are not expected to give rise to highenergy emission. In contrast to early-type stars they do not drive strong stellar winds, in which instabilities arise that can be responsible for the X-rays observed from O- and early B-type stars (Owocki \& Cohen 1999; Lucy \& White 1980). The empirical relation between the bolometric and the X-ray luminosity observed for hot stars breaks down near spectral type $\sim$ B2 (Berghöfer et al. 1996, hereafter BSC96), approximately coincident with the transition from a strong-wind to a weak-wind regime (Babel 1996). Stars with cooler spectral types show a large scatter of $\mathrm{X}$-ray luminosity for given bolometric luminosity.

Intermediate-mass stars also do not possess convection zones necessary to drive a stellar dynamo. Therefore, magnetic activity that generates X-ray emission of late-type stars (Parker 1955, 1993; Rüdiger \& Brandenburg 1995) seems not to be a viable alternative. The minimum depth of a convective envelope able to support magnetic activity is not well established. Based on studies of the X-ray detection rate as a function of color for mainsequence (MS) stars the onset of significant dynamo action is

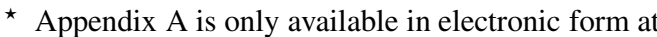
http://www . edpsciences.org
}

placed somewhere near spectral type A7 (Schmitt et al. 1985; Hünsch 2001).

As outlined above, obvious qualitative changes in the properties of the observed X-ray emission coincide with the approximate break-down of known emission mechanisms. Nevertheless, contrary to the expectation a relatively large number of A- and B-type stars apparently are not X-ray dark, with hundreds of them listed in the catalogs of X-ray sources detected during the ROSAT All-Sky Survey (RASS); see Berghöfer et al. (1996); Hünsch et al. (1998). In the absence of a physical explanation, the X-ray emission of late-B and early-A stars is commonly attributed to unresolved late-type companions. The contraction timescale of late-type pre-MS stars to the MS is comparable to the mean lifetime of late B-type stars on the MS. Hence, the hypothesized companions of a MS B-type star must be young late-type stars approaching the MS, and - because of the relation between X-ray emission and stellar youth - they are by nature strong X-ray sources. Based on this argument Lindroos (1986) has isolated 78 pairs composed of MS early-type star and a likely physically bound pre-MS star. These systems are henceforth termed 'Lindroos systems'.

Testing the hypothesis that unresolved companions produce the observed X-rays from A- and B-type stars requires highspatial resolution observations in the optical/IR and the X-ray band. The first step in this approach is to search for companions within the X-ray error box of X-ray detected A/B-stars. The preferred wavelength is the near-IR, where the contrast between the intermediate-mass star and an eventual cool companion is 
lower than in the optical, and where the adaptive optics (AO) technique allows to obtain diffraction limited images with subarcsecond separations. In this way Hubrig et al. (2001) reported the discovery of new companions for 19 of 49 B-type stars selected among the X-ray emitters detected during the RASS. The separations between the new IR objects and the B-type primary range between $0.2-14^{\prime \prime}$. The IR photometry of these new objects was used to place them in the HR diagram, estimating their masses and spectral types. Both are consistent with them being late-type stars. Therefore, they are good candidates for the origin of the X-ray emission.

After the identification of the companions, the claim that they are responsible for the observed X-ray emission must be proven by resolving them from the intermediate-mass stars in $\mathrm{X}$-rays. So far, observational investigations of this issue have been restricted to the few known A- and B-type stars with companions at separations large enough to be resolved by the low spatial resolution X-ray instruments available. A sample of eight visual binaries with separations $>10^{\prime \prime}$ was examined by Berghöfer \& Schmitt (1994) using the ROSAT High Resolution Imager (HRI). But in only one case could the X-ray emission be ascribed to the known visual late-type companion. On the other hand, a ROSAT HRI study of the Lindroos systems has shown that both the primaries - mostly of spectral type B - and their late-type companions emit X-rays at similar levels (Schmitt et al. 1993; Huélamo et al. 2000). This was taken as support for the hypothesis that the X-ray emission from late B-type stars in fact originates from closer pre-MS late-type companions unresolvable by the ROSAT HRI.

Chandra is the first X-ray satellite that allows for a spatial resolution that comes near to that in the IR. Systems as close as $\sim 1^{\prime \prime}$ can be studied. At the distance of 100-200 pc, where many of the X-ray emitting B- and A-type stars are located, this corresponds to $<200 \mathrm{AU}$ separation. This is much smaller than the maximum separation of visual binaries in the solar neighborhood (see Close et al. 1990; Duquennoy \& Mayor 1991). Therefore, it is plausible to consider the faint objects found at a separation of a few arc-seconds from nearby B- and A-type stars bound companions, and not just chance projections. However, for this study (i.e. the investigation of the origin of X-rays from B-type stars) this question is not of great importance.

We have performed a series of Chandra imaging observations pointing at multiple stars with a B-type primary selected from recent AO surveys. The present article is a continuation of the work presented by Stelzer et al. (2003) (henceforth SHH03). In Sect. 2 we explain the selection of the sample observed with Chandra. In Sect. 3 the observations and the data analysis are described. The results from source detection and the X-ray properties of the detections are also presented in Sect. 3, including a discussion of the X-ray lightcurves and spectra. We discuss the $L_{\mathrm{x}} / L_{\text {bol }}$ relation in Sect 4. In Sect. 5 the results are put in the context of possible emission mechanisms, and Appendix A has information on the individual targets.

\section{The sample}

Our project consists of Chandra snapshots of late-B type stars detected in the RASS and known to have close faint companions revealed in recent near-IR AO observations. Results for the first 5 objects were presented by SHH03, where our selection criteria have been described in detail. Briefly summarized, the targets must (i) have been detected previously in low-spatial resolution X-ray observations, (ii) have companions identified by recent $\mathrm{AO}$ observations and unknown at the time of the previous
X-ray observations, (iii) have separations ranging from $1-8^{\prime \prime}$ between the B/A star and the AO companion (to be resolvable with Chandra but no other presently or previously available X-ray instrument), and (iv) the primaries show no signs of intrinsic binarity according to the Hipparcos data base (The Hipparcos Catalogue, 1997, ESA SP-1200) and the $\Delta \mu$ data base (Wielen et al. 2000).

In this paper the sample presented by $\mathrm{SHHO3} \mathrm{is} \mathrm{comple-}$ mented by Chandra observations of another 5 late-B stars. Our targets were chosen from the work of Hubrig et al. (2001). We have added to our sample one further intermediate-mass star from the Chandra archive: HD 129791 is a Lindroos system composed of a A0 star on the MS and a K5 companion at 35". One of our other targets, HD 32964, is also a Lindroos system, i.e. this star has both a wide companion at $~ 53^{\prime \prime}$ (the Lindroos secondary) and a close companion discovered by Hubrig et al. (2001).

The separations of the two Lindroos secondaries are large enough that they have been resolved with ROSAT. Therefore, these companions cannot provide the explanation of the X-ray emission from the B-type primaries. However, studying their X-ray emission may be instructive for two reasons: (1) It may help to discriminate between them being physically bound companions and unbound chance projections. This is, because - as explained in Sect. 1 - true late-type companions to B stars on the MS are still in their pre-MS contraction phase where they are strongly magnetically active, while unrelated old foreground or background stars are not expected to be X-ray bright. (2) It allows us to search for differences between primaries and secondaries that could indicate different emission processes.

Thus, the new sample we present in this paper comprises 6 targets, 5 from our dedicated Chandra program and 1 from the Chandra archive. In Table 1 some information on the targets is given. Columns 1-5 give the designation, the Hipparcos position of the primary, distance, and spectral type. The separation, position angle, an identifier, and the reference of all known companions are listed in Cols. 6-9. We use "L" for Lindroos companions, and " $\mathrm{B}$ " and " $\mathrm{C}$ " for further visual companions. The last columns of Table 1 give the Chandra observation identification number, instrument and exposure time. Throughout this paper we also use results from the first part of our survey (SHH03). The targets presented by SHH03 are also listed in Table 1. Where necessary, the X-ray properties of that group of stars have been re-examined to yield a consistent description of the whole sample.

True companionship has not been established for any of the new IR objects in our sample, and only for some of the Lindroos secondaries. Confirmation that they are true companions and not just chance projections requires observations of their proper motion and/or spectra. In absence of definite information about their status we will for simplicity continue to call the IR objects "companions" and the B-type stars "primaries".

\section{Observations and data analysis}

Our original targets were observed with the standard imaging array of the Advanced CCD Imaging Spectrometer (ACIS-I) with a nominal frame time of $3.2 \mathrm{~s}$. The object added from the archive, HD 129791, had been placed on an ACIS-S chip. ACIS-S provides higher sensitivity than ACIS-I for soft energies. However, its limiting optical magnitude is fainter. Therefore, in order to avoid the risk of contamination by optical light from the optically bright primary star the frame time was reduced to $0.9 \mathrm{~s}$. The net exposure time per target was $\sim 2-8 \mathrm{ks}$. 
Table 1. Target list of Chandra observed late-B and early-A stars: position, distance, and spectral type; separation, position angle and an identifier of companion candidates; Chandra observation ID, detector ID and exposure time.

\begin{tabular}{|c|c|c|c|c|c|c|c|c|c|c|c|}
\hline \multirow[t]{2}{*}{ Designation } & \multicolumn{2}{|c|}{ Position $^{a}$} & \multirow{2}{*}{$\begin{array}{r}\text { Dist }^{a} \\
{[\mathrm{pc}]}\end{array}$} & \multirow[t]{2}{*}{$\mathrm{SpT}^{b}$} & \multirow{2}{*}{$\begin{array}{c}\mathrm{Sep}^{c} \\
{\left[{ }^{\prime \prime}\right]} \\
\end{array}$} & \multirow{2}{*}{$\begin{array}{c}\mathrm{PA}^{c} \\
{\left[{ }^{\circ}\right]}\end{array}$} & \multirow[t]{2}{*}{ Comp. } & \multirow[t]{2}{*}{ Ref. } & \multicolumn{3}{|c|}{ ACIS observations } \\
\hline & $\alpha_{2000}$ & $\delta_{2000}$ & & & & & & & ObsID & Instr & Expo [s] \\
\hline \multicolumn{12}{|c|}{ New Targets } \\
\hline HD 32964 & 05:06:45.67 & $-04: 39: 17.4$ & 86 & B9 & $\begin{array}{l}1.61 \\
52.8\end{array}$ & $\begin{array}{r}307.1 \\
10\end{array}$ & $\begin{array}{l}\text { B } \\
\text { L }\end{array}$ & $\begin{array}{c}(1 *) \\
(2)\end{array}$ & 3739 & ACIS-I & 2518 \\
\hline HD 73952 & 08:38:44.96 & $-53: 05: 26.1$ & 155 & B8 & 1.16 & 205.3 & $\mathrm{~B}$ & (1) & 3740 & ACIS-I & 7630 \\
\hline HD 110073 & $12: 39: 52.56$ & $-39: 59: 14.8$ & 109 & B8 & 1.19 & 75.0 & B & (1) & 3741 & ACIS-I & 3241 \\
\hline HD 129791 & $14: 45: 57.66$ & $-44: 52: 02.9$ & 130 & A0 & 35.3 & 205.5 & $\mathrm{~L}$ & (2) & 0627 & ACIS-S & 6584 \\
\hline HD 134837 & $15: 13: 07.69$ & $-36: 05: 28.8$ & 111 & B8 & 4.70 & 154.3 & B & (1) & 3742 & ACIS-I & 2929 \\
\hline HD 134946 & $15: 13: 17.44$ & $-24: 00: 30.2$ & 126 & B8 & 8.21 & 45.3 & B & (1) & 3743 & ACIS-I & 2335 \\
\hline \multicolumn{12}{|c|}{ Stars from Stelzer et al. (2003) } \\
\hline HD 1685 & $00: 20: 39.03$ & $-69: 37: 29.7$ & 94 & B9 & 2.28 & 211.4 & B & (1) & 2541 & ACIS-I & 2338 \\
\hline HD 113703 & 13:06:16.70 & $-48: 27: 47.8$ & 127 & B5 & $\begin{array}{l}1.55 \\
11.5\end{array}$ & $\begin{array}{r}268.2 \\
79\end{array}$ & $\begin{array}{l}\text { B } \\
\text { L }\end{array}$ & $\begin{array}{l}\text { (3) } \\
(4)\end{array}$ & 0626 & ACIS-S & 12184 \\
\hline HD 123445 & $14: 08: 51.89$ & $-43: 28: 14.8$ & 218 & B9 & $\begin{array}{l}5.56 \\
5.38 \\
28.6\end{array}$ & $\begin{array}{r}65.0 \\
64.0 \\
35\end{array}$ & $\begin{array}{l}B \\
C \\
L\end{array}$ & $\begin{array}{l}(5) \\
(5) \\
(2)\end{array}$ & 2542 & ACIS-I & 2237 \\
\hline HD 133880 & $15: 08: 12.12$ & $-40: 35: 02.1$ & 126 & B8 & 1.22 & 109.2 & $\mathrm{~B}$ & (1) & 2543 & ACIS-I & 2461 \\
\hline HD 169978 & 18:31:22.43 & $-62: 16: 41.9$ & 147 & B7.5 & 3.09 & 168.7 & $\mathrm{~B}$ & $(1)$ & 2544 & ACIS-I & 2420 \\
\hline
\end{tabular}

${ }^{a}$ Hipparcos position and distance for the A/B-type star.

${ }^{b}$ Spectral types adopted from the SIMBAD database at http://simbad.u-strasbg.fr/Simbad

${ }^{c}$ Separations have been measured in the detector space. References: $(1)$ - Hubrig et al. (2001), $\left(1^{*}\right)$ - PA redetermined on the ADONIS image, (2) - Turon et al. (1993), (3) - Shatsky \& Tokovinin (2002), (4) - Huélamo et al., in prep., (5) - Huélamo et al. (2001).

The data analysis was carried out using the CIAO software package ${ }^{1}$ version 3.2 in combination with the calibration database (CALDB) version 3.0.0. We started our analysis with the level 1 events file provided by the pipeline processing at the Chandra X-ray Center (CXC). On all observations processed at the CXC with CALDB version earlier than 2.9 we applied a new gain map and updates on the geometry (focal length, ACIS pixel size and chip positions). For observations processed with a later version of CALDB these modifications were performed automatically during the pipeline process. In the process of converting the level 1 events file to a level 2 events file for each of the observations we performed the following steps: A correction for the charge transfer inefficiency (CTI) was applied for data with processing version earlier than 6.12 , i.e. for those where the CTI correction was not yet part of the standard pipeline processing at the CXC. We removed the pixel randomization which is automatically applied by the CXC pipeline in order to optimize the spatial resolution. We filtered the events file for event grades (retaining the standard $A S C A$ grades 0, 2, 3, 4 and 6), and applied the standard good time interval file. Events flagged as cosmic ray afterglow were retained after inspection of the images revealed that a substantial number of source photons erroneously carry this flag. Since the positional accuracy is particularly important for our observations we also checked the astrometry for any known systematic aspect offsets using the CIAO aspect calculator $^{2}$. Small offsets are present in some of the observations,

${ }^{1}$ CIAO is made available by the $\mathrm{CXC}$ and can be downloaded from http://asc.harvard.edu/ciao/download

2 See http://asc.harvard.edu/cal/ASPECT/

fix_offset/fix_offset.cgi and the aspect was corrected accordingly by modifying the respective header keywords in the events level 2 file.

\subsection{Source detection and identification}

Source detection was carried out with the wavdetect algorithm (Freeman et al. 2002). This algorithm correlates the data with a Mexican hat function to search for deviations from the background. The wavdetect mechanism is well suited to separate closely spaced point sources. We used wavelet scales between 1 and 8 in steps of $\sqrt{2}$. The size of the analysed images is $50 \times$ 50 sky pixels $\left(1\right.$ sky pixel $\left.=0.492^{\prime \prime}\right)$. For exceptions with known companions at wider separations the source detection was performed in a slightly larger area. The threshold for the significance of the detection was set to $2 \times 10^{-7}$. For this value the detection of one spurious source is expected in a $2048 \times 2048$ pixel wide image.

The identification of all X-ray sources with components of our target systems is summarized in Cols. $1-3$ of Table 4 . We defer a discussion of Table 4 to Sect. 3.3. Source identification was done by measuring the distance $\left(\Delta_{\mathrm{ox}}\right)$ between each X-ray source and the optical/IR positions of all known visual components in the system. Then we assigned each X-ray source to the closest of the optical/IR objects. This procedure turned out to be unambiguous for all spatially resolved components. No limit was put on the maximum acceptable $\Delta_{\mathrm{ox}}$, but in practice these offsets are small (typically within $0.2^{\prime \prime}$ ). As a rule, source photons were extracted from a circle centered on the wavdetect source position within the radius that includes $90 \%$ of the point-spread-function (PSF) at $1.5 \mathrm{keV}$, depending on the position of the source on 


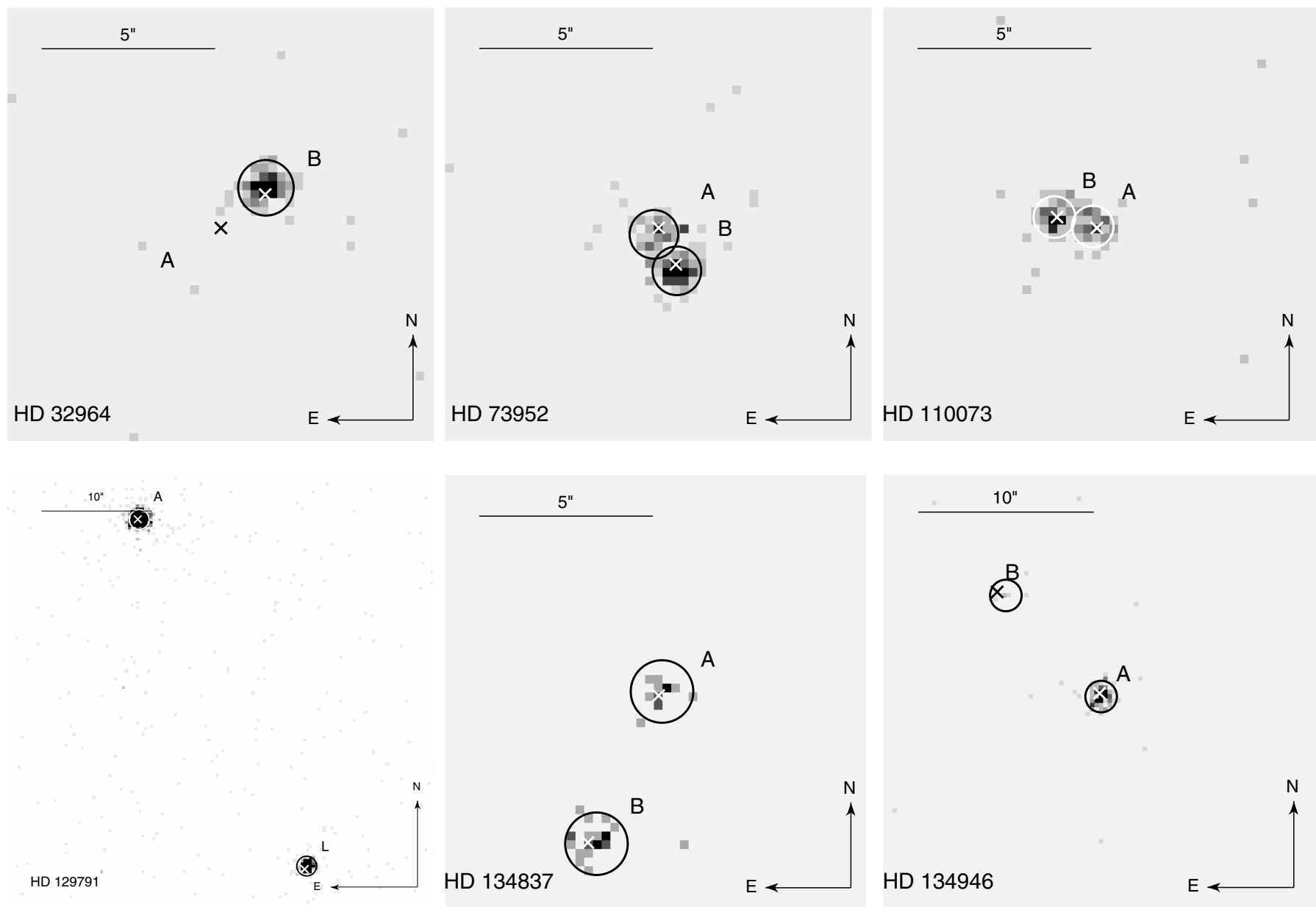

Fig. 1. Chandra ACIS images of B/A-type stars and faint companions. The size of the images is $25 \times 25$ pix with pixel size of 0.25 ", except for HD $129791(80 \times 80$ pix $)$ and HD $134946(50 \times 50$ pix $)$. Crosses denote the optical/IR position of the individual components in the multiple system, circles mark the photon extraction areas centered on the position of X-ray sources detected with wavdetect. The primaries are labeled "A", the companions with letters "B" and "L", where "L" stands for "Lindroos companion".

the detector. For sources that are not fully resolved we defined smaller non-overlapping photon extraction circles.

\subsection{Results of source detection}

Figure 1 shows a portion of the ACIS images around our targets. We overplot the photon extraction areas (circles), as well as the Hipparcos position of the primary and the IR position of the companions (x-shaped symbols). Surprisingly, in the new sample X-rays are detected from all but one of the primaries. Similar to the findings by $\mathrm{SHH} 03$ most companions are also X-ray emitters, yielding 11 new X-ray sources, and a total of 19 X-ray sources when combined with the targets of SHHO3.

Table 2 gives the published stellar parameters of the primaries, a summary of the X-ray detections, and a flag if there are known companions that remained unresolved with Chandra. Among the 7 (out of 11) detected primaries two are known to have unresolved companions: HD 169978 is a spectroscopic binary (see discussion in SHH03), and HD 110073 is listed as an SB 1 in the catalog of Ap stars by Schneider (1981). However, it can not be excluded that there are close unresolved companions among the other primaries, because to date no systematic observations for spectroscopic companions have been carried out for these objects.

Concerning the resolved components, the X-ray detection can help to single out true companions from chance projections.
Table 2. Stellar parameters for B- and A-type primaries observed with Chandra. The last two columns provide flags indicating whether the star is detected in X-rays ("X") and whether it has known companions not resolved with Chandra ("C").

\begin{tabular}{lrrrrr|ll}
\hline \hline Name & $\begin{array}{r}\log T_{\text {eff }} \\
{[\mathrm{K}]}\end{array}$ & $\log \frac{L_{\text {bol }}}{L_{\odot}}$ & Ref. & $\begin{array}{r}v \sin i \\
{[\mathrm{~km} / \mathrm{s}]}\end{array}$ & Ref. & $\mathrm{X}$ & $\mathrm{C}$ \\
\hline HD 32964 & 4.05 & 2.34 & $(1)$ & 30 & $(5)$ & - & $\sqrt{ }$ \\
HD 73952 & 4.09 & 2.10 & $(1)$ & & & $\sqrt{ }$ & \\
HD 110073 & 4.11 & 2.59 & $(1)$ & 28 & $(6)$ & $\sqrt{ }$ & $\sqrt{ }$ \\
HD 129791 & 4.01 & 1.57 & $(2,3)$ & 280 & $(7)$ & $\sqrt{ }$ & \\
HD 134837 & 4.10 & 1.97 & $(1)$ & & & $\sqrt{ }$ & \\
HD 134946 & 4.14 & 2.19 & $(1)$ & 150 & $(5)$ & $\sqrt{ }$ & \\
\hline HD 1685 & 4.02 & 1.87 & $(1)$ & 236 & $(7)$ & $\sqrt{ }$ \\
HD 113703 & 4.19 & 2.67 & $(4)$ & 216 & $(6)$ & - & \\
HD 123445 & 4.02 & 2.25 & $(4)$ & 66 & $(7)$ & - & \\
HD 133880 & 4.08 & 2.20 & $(1)$ & & & - & \\
HD 169978 & 4.11 & 2.82 & $(1)$ & 91 & $(6)$ & $\sqrt{ }$ & $\sqrt{ }$ \\
\hline
\end{tabular}

(1) - Hubrig et al. (2001), (2) - de Geus et al. (1989), (3) - Gerbaldi et al. (2001), (4) - this paper: $L_{\mathrm{bol}}$ from $V$ mag, $T_{\text {eff }}$ from spectral type with conversion by Kenyon \& Hartmann (1995), (5) - Abt et al. (2002), (6) - Uesugi \& Fukuda (1970), (7) - Royer et al. (2002).

This is related to the argument laid out in the introduction, that B-type stars on the MS are young, such that bound late-type companions are on the pre-MS where they are naturally strong $\mathrm{X}$-ray emitters. It is well established that $\mathrm{T}$ Tauri stars have 


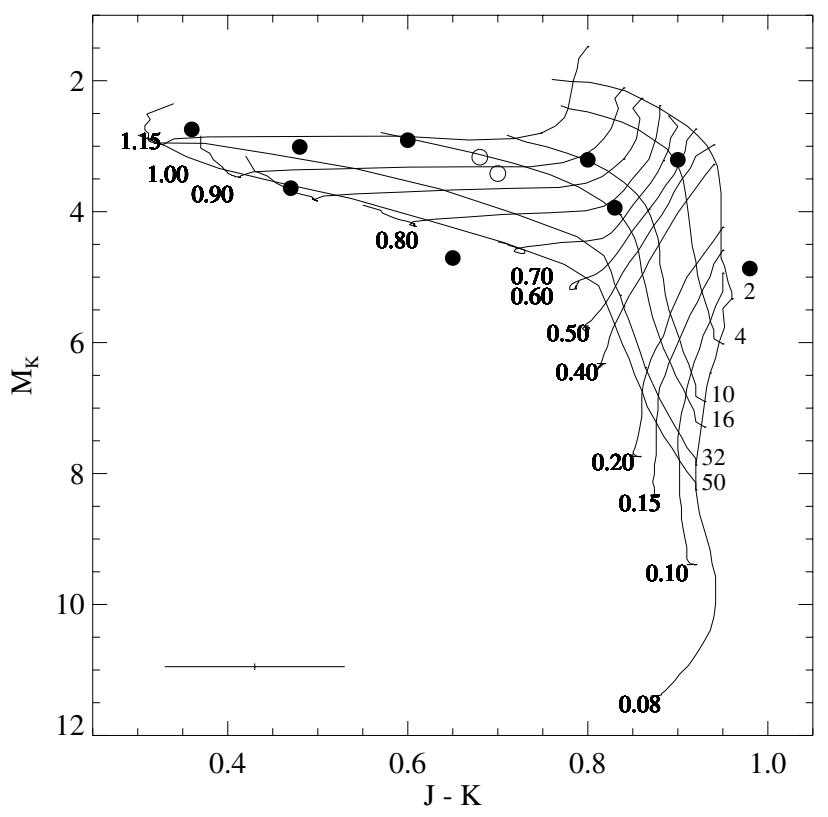

Fig. 2. Near-IR color-magnitude diagram for companion candidates to intermediate-mass stars. filled symbols - objects detected with Chandra, open symbols - objects not detected with Chandra. The grid represents pre-MS models from Baraffe et al. (1998). For clarity errors are not shown, but are typically $0.1 \mathrm{mag}$ (see error bar on the bottom left), such that all objects shown in this diagram are compatible with being on the pre-MS.

high X-ray luminosities, albeit with a large spread: $\log L_{\mathrm{x}} \sim$ 28...31 erg/s (e.g. Stelzer \& Neuhäuser 2001; Preibisch et al. 2005). For the same range of bolometric luminosity nearby field stars display much lower activity levels, with typically $\log L_{\mathrm{x}} \sim 26 \ldots 28 \mathrm{erg} / \mathrm{s}$ Schmitt \& Liefke (2004); see also Fig. 6 of Preibisch et al. (2005) for a direct comparison to T Tauri stars.

Of the 15 companions of the 11 B-type stars observed with Chandra 12 are detected, and most of them show X-ray luminosities in the range $10^{29 . .30} \mathrm{erg} / \mathrm{s}$ (see Table 4 and Sect. 3.3), yielding strong evidence for them being pre-MS stars. However, final classification requires confirmation of the companion status by means of spectroscopy (optical spectroscopy should reveal a $\mathrm{Li}$ I absorption feature at $6708 \AA$ indicating their pre-MS nature) or proper motion. At present this information is available for only a minority of the companions discussed in this article. Available near-IR photometry allows for a rough estimate of their evolutionary stage, comparing their position in the near-IR color-magnitude diagram (CMD) to pre-MS models.

Figure 2 shows the $M_{\mathrm{K}}$ vs. $J-K$ diagram with model calculations by Baraffe et al. (1998): $Y=0.275,[M / H]=0, \alpha_{\mathrm{ML}}=1$. The positions of all companion candidates with published $J$ and $K$ magnitudes (summarized in Table 3 ) are overplotted. For the distances needed to compute the absolute $K$-band magnitude we assumed that all companions are bound to the primaries. Note that three of the presumed low-mass companions discovered in AO observations have been observed only in the $K$-band, such that they cannot be placed in Fig. 2. For the Lindroos companions we extract the near-IR photometry from the $2 \mathrm{MASS}^{3}$ catalog. Only one companion (HD 1685 B) has near-IR colors that are incompatible with the location of pre-MS stars in the CMD, and therefore this object is likely physically unrelated to the Btype star. For the undetected Lindroos companions of HD 32964

3 The 2 MASS All Sky Catalog of point sources is available online at http://vizier.u-strasbg.fr/viz-bin/VizieR and HD 123445, the near-IR photometry is compatible with the pre-MS models. The upper limits for their X-ray luminosities are between $10^{28 \ldots 29} \mathrm{erg} / \mathrm{s}$, in the range where field stars and pre-MS stars overlap, such that their nature remains unclear.

We tentatively assigned a companion status to each object based on X-ray detection/non-detection and near-IR photometry. Corresponding flags are given in the last two columns of Table 3. Thus, for the majority of companions both X-rays and near-IR data consistently indicate youth.

\subsection{X-ray properties}

In Table 4 we list the X-ray parameters of all known components, whether detected or not. We give the HD number of the target (Col. 1), component identifier (Col. 2), and a flag that distinguishes X-ray detections ("V") from non-detections ("-_) (Col. 3). The offset between X-ray and optical/IR position and the significance of detection resulting from the wavdetect algorithm are given in Cols. 4 and 5. The number of counts (Col. 6) refers to the $0.5-8 \mathrm{keV}$ passband, and comprises a fraction of between 35 and $90 \%$ of the total source counts (see Col. 9), depending on the extraction radius. To compute upper limits for the undetected components of our target systems we used the method for Poisson-distributed counting data described by Kraft et al. (1991). We took account of the background fluctuations by estimating the background within a squared area of $1^{\prime}$ side length centered on the optical/IR position of the respective star but excluding all detected sources, and scaling this mean background to the source extraction area. Columns 7 and 8 of Table 4 show hardness ratios defined as $H R=(H-S) /(H+S)$, where $H$ and $S$ are the number of counts in a hard band and in a soft band, respectively. $H R 1$ is defined using emission in the $0.5-1 \mathrm{keV}(S)$ and the $1-8 \mathrm{keV}(H)$ bands, and $H R 2$ from the $1-2 \mathrm{keV}(S)$ and the $2-8 \mathrm{keV}(H)$ band. In the final columns of Table 4 we list the PSF fraction included in the source extraction area, the PSF- and absorption-corrected broad-band X-ray luminosity and the ratio of X-ray to bolometric luminosity. The X-ray luminosities have been computed with PIMMS assuming an iso-thermal emitting plasma with $k T=1 \mathrm{keV}$. Absorption is neglected, except for HD 129791 where a column density of $N_{\mathrm{H}}=5.0 \times 10^{20} \mathrm{~cm}^{-2}$ is assumed, corresponding to the value derived from the optical extinction $\left(A_{\mathrm{V}}=0.26 \mathrm{mag}\right.$; Ryter 1996). In general, stellar coronae are characterized by a multi-temperature plasma. Therefore, the assumption of a 1-T model may not be appropriate, and consequently the X-ray luminosities may not be very precise. In Sect. 3.5 more reliable X-ray luminosities are derived directly from the X-ray spectrum for the brighter sources.

\subsection{X-ray lightcurves}

We binned lightcurves for all detected sources in $400 \mathrm{~s}$ intervals. Obviously, the detection of variability requires both high statistics and a good time resolution, and our choice of the binning is a compromise between these two opposing effects. The duration of these snapshop observations $(\sim 2-12 \mathrm{ks})$ is well below typical variability time-scales on active stars, and therefore we do not expect to detect much variability. Indeed, a KS-test yields only one of the $19 \mathrm{X}$-ray sources in the sample variable at $>99 \%$ confidence level (HD 73952 B). The lightcurve of this object is displayed in Fig. 3, with a sharp increase in the count rate about two thirds into the observation.

The occurrence of 1 flare in $\sim 80 \mathrm{ks}$ (the sum of the observing time of all detected stars in the total sample) is not unusual; it is 
Table 3. Near-IR photometry and stellar parameters for known companion candidates. The last two columns provide flags for the companion status: " $\sqrt{ }$ " - X-ray detection or near-IR photometry suggests late-type pre-MS star and consequently likely bound system, "?" - not resolved in $\mathrm{X}$-rays or no near-IR color available.

\begin{tabular}{lrrrrrrrc}
\hline \hline HD & $\begin{array}{c}J \\
{[\mathrm{mag}]}\end{array}$ & $\begin{array}{c}K \\
{[\mathrm{mag}]}\end{array}$ & Ref. & $\begin{array}{r}\log T_{\text {eff }} \\
{[\mathrm{K}]}\end{array}$ & $\log \frac{L_{\text {bol }}}{L_{\odot}}$ & Ref. & \multicolumn{2}{c}{ Companionship } \\
X-rays & NIR-phot. \\
\hline HD 32964 L & 8.79 & 8.09 & $(3)$ & 3.63 & -0.18 & $(5)$ & - & $\sqrt{ }$ \\
HD 32964 B & 10.03 & 9.38 & $(1)$ & 3.64 & -0.94 & $(1)$ & $\sqrt{ }$ & $\sqrt{ }$ \\
HD 73952 B & 11.8 & 10.82 & $(1)$ & 3.61 & -1.06 & $(1)$ & $\sqrt{ }$ & $\sqrt{ }$ \\
HD 129791 L & 10.37 & 9.54 & $(3)$ & 3.65 & -0.75 & $(6)$ & $\sqrt{ }$ & $\sqrt{ }$ \\
HD 110073 B & 8.29 & 7.93 & $(1)$ & 3.75 & +0.09 & $(1)$ & $\sqrt{ }$ & $\sqrt{ }$ \\
HD 134837 B & - & 10.99 & $(1)$ & 3.51 & -1.55 & $(1)$ & $\sqrt{ }$ & $?$ \\
HD 134946 B & - & 12.51 & $(1)$ & 3.43 & -2.18 & $(1)$ & $\sqrt{ }$ & $?$ \\
\hline HD 113703 B & 9.63 & 9.16 & $(2)$ & & -0.32 & $(2)$ & $\sqrt{ }$ & $\sqrt{ }$ \\
HD 113703 L & 9.01 & 8.53 & $(3)$ & 3.68 & -0.38 & $(6)$ & $\sqrt{ }$ & $\sqrt{ }$ \\
HD 1685 B & 11.7 & 10.1 & $(1)$ & 3.59 & -1.20 & $(1)$ & $\sqrt{ }$ & - \\
HD 123445 L & 10.54 & 9.86 & $(3)$ & 3.65 & -0.81 & $(6)$ & - & $\sqrt{ }$ \\
HD 123445 B & 10.8 & 9.9 & $(4)$ & & -0.49 & $(4)$ & $?$ & $\sqrt{ }$ \\
HD 123445 C & 10.7 & 9.9 & $(4)$ & & -0.36 & $(4)$ & $?$ & $\sqrt{ }$ \\
HD 133880 B & 9.01 & 8.41 & $(1)$ & 3.76 & +0.15 & $(1)$ & $\sqrt{ }$ & $\sqrt{ }$ \\
HD 169978 B & - & 12.69 & $(1)$ & 3.50 & -2.40 & $(1)$ & - & $?$ \\
\hline
\end{tabular}

(1) - Hubrig et al. (2001), (2) - Shatsky \& Tokovinin (2002), (3) - Cutri et al. (2003), (4) - Huélamo et al. (2001), (5) - Lindroos (1985), (6) Lindroos (1986).

Table 4. X-ray parameters of all components in the sample; see text in Sect. 3.3.

\begin{tabular}{|c|c|c|c|c|c|c|c|c|c|c|}
\hline Designation & $\mathrm{Opt} / \mathrm{IR}$ & X-rays & $\begin{array}{c}\Delta_{\mathrm{xo}} \\
{\left[{ }^{\prime \prime}\right]}\end{array}$ & Sign. & Counts* & HR 1 & HR 2 & $\begin{array}{c}\text { PSF } \\
{[\%]}\end{array}$ & $\begin{array}{c}\log L_{\mathrm{x}}^{*} \\
{[\mathrm{erg} / \mathrm{s}]}\end{array}$ & $\log \left(\frac{L_{\mathrm{x}}^{*}}{L_{\mathrm{bol}}}\right)$ \\
\hline \multirow{3}{*}{ HD 32964} & A & - & - & - & $<8.2$ & - & - & 0.88 & $<28.2$ & $<-7.4$ \\
\hline & B & $\sqrt{ }$ & 0.20 & 43.9 & $99.0 \pm 11.0$ & $0.15 \pm 0.16$ & $-0.72 \pm 0.22$ & 0.88 & 29.3 & -3.4 \\
\hline & $\mathrm{L}$ & - & - & - & $<4.4$ & - & - & 0.90 & $<27.9$ & $<-5.5$ \\
\hline \multirow[t]{2}{*}{ HD 73952} & A & $\sqrt{ }$ & 0.23 & 16.1 & $43.0 \pm 7.6$ & $-0.02 \pm 0.27$ & $-0.90 \pm 0.38$ & 0.83 & 29.0 & -6.7 \\
\hline & B & $\sqrt{ }$ & 0.19 & 39.0 & $92.0 \pm 10.6$ & $0.22 \pm 0.17$ & $-0.54 \pm 0.23$ & 0.83 & 29.3 & -3.2 \\
\hline \multirow[t]{2}{*}{ HD 110073} & A & $\sqrt{ }$ & 0.13 & 16.0 & $28.0 \pm 6.4$ & $-0.21 \pm 0.35$ & $-0.45 \pm 0.68$ & 0.77 & 29.0 & -7.1 \\
\hline & B & $\sqrt{ }$ & 0.08 & 20.5 & $45.0 \pm 7.8$ & $-0.02 \pm 0.26$ & $-0.91 \pm 0.37$ & 0.77 & 29.3 & -4.4 \\
\hline \multirow[t]{2}{*}{ HD 134837} & A & $\sqrt{ }$ & 0.15 & 6.2 & $12.0 \pm 4.6$ & $0.00 \pm 0.60$ & $-0.67 \pm 1.02$ & 0.90 & 28.5 & -7.0 \\
\hline & B & $\sqrt{ }$ & 0.25 & 8.1 & $22.0 \pm 5.8$ & $0.00 \pm 0.40$ & $-0.64 \pm 0.65$ & 0.90 & 28.8 & -3.3 \\
\hline \multirow[t]{2}{*}{ HD 134946} & A & $\sqrt{ }$ & 0.20 & 57.2 & $152.0 \pm 13.4$ & $0.13 \pm 0.13$ & $-0.81 \pm 0.17$ & 0.90 & 29.8 & -6.0 \\
\hline & B & $\sqrt{ }$ & 0.53 & 1.6 & $5.0 \pm 3.4$ & $-0.20 \pm 1.17$ & $-1.00 \pm 3.09$ & 0.90 & 28.3 & -3.1 \\
\hline \multirow[t]{2}{*}{ HD 129791} & A & $\sqrt{ }$ & 0.13 & 236.6 & $1471.0 \pm 39.4$ & $-0.14 \pm 0.04$ & $-0.77 \pm 0.06$ & 0.90 & 30.3 & -4.9 \\
\hline & $\mathrm{L}$ & $\sqrt{ }$ & 0.33 & 101.2 & $305.0 \pm 18.5$ & $-0.30 \pm 0.08$ & $-0.85 \pm 0.14$ & 0.90 & 29.6 & -3.3 \\
\hline \multirow[t]{3}{*}{ HD 113703} & A & - & - & - & $<17.5$ & - & - & 0.68 & $<28.1$ & $<-8.2$ \\
\hline & B & $\sqrt{ }$ & 0.21 & 228.4 & $1257.0 \pm 36.5$ & $-0.34 \pm 0.04$ & $-0.77 \pm 0.07$ & 0.90 & 29.8 & -3.5 \\
\hline & $\mathrm{L}$ & $\sqrt{ }$ & 0.43 & 341.2 & $2943.0 \pm 55.3$ & $-0.12 \pm 0.03$ & $-0.67 \pm 0.04$ & 0.90 & 30.2 & -3.0 \\
\hline \multirow[t]{3}{*}{ HD 1685} & A & $\sqrt{ }$ & 0.20 & 17.1 & $42.0 \pm 7.5$ & $0.00 \pm 0.27$ & $-0.90 \pm 0.38$ & 0.90 & 29.0 & -6.5 \\
\hline & B & $\sqrt{ }$ & 0.29 & 27.7 & $66.0 \pm 9.2$ & $0.03 \pm 0.21$ & $-0.65 \pm 0.31$ & 0.90 & 29.2 & -3.2 \\
\hline & - & $\sqrt{ }$ & - & 2.6 & $5.0 \pm 3.4$ & $0.20 \pm 1.17$ & $0.33 \pm 2.00$ & 0.90 & 28.1 & - \\
\hline \multirow[t]{4}{*}{ HD 123445} & $\mathrm{~A}$ & - & - & - & $<4.4$ & - & - & 0.90 & $<28.8$ & $<-7.1$ \\
\hline & $\mathrm{B}$ & $\sqrt{ }$ & 0.49 & 27.2 & $59.0 \pm 8.7$ & $0.05 \pm 0.22$ & $-0.81 \pm 0.31$ & 0.90 & 29.9 & -3.2 \\
\hline & $\mathrm{C}$ & "I & 0.46 & $\prime \prime$ & $\prime \prime$ & $\prime \prime$ & $\prime \prime$ & $\prime \prime$ & $\prime \prime$ & -3.3 \\
\hline & $\mathrm{L}$ & - & - & - & $<4.4$ & - & - & 0.90 & $<28.8$ & $<-4.4$ \\
\hline \multirow[t]{2}{*}{ HD 133880} & $\mathrm{~A}$ & - & - & - & $<8.2$ & - & - & 0.35 & $<28.9$ & $<-6.9$ \\
\hline & $\mathrm{B}$ & $\sqrt{ }$ & 0.24 & 64.2 & $193.0 \pm 14.9$ & $0.16 \pm 0.11$ & $-0.55 \pm 0.15$ & 0.90 & 29.9 & -3.8 \\
\hline \multirow[t]{2}{*}{ HD 169978} & $\mathrm{~A}$ & $\sqrt{ }$ & 0.15 & 47.1 & $122.0 \pm 12.1$ & $0.02 \pm 0.15$ & $-0.65 \pm 0.21$ & 0.90 & 29.8 & -6.6 \\
\hline & B & - & - & - & $<14.6$ & - & - & 0.90 & $<28.9$ & $<-2.3$ \\
\hline
\end{tabular}

${ }^{*}$ In the $0.5-8 \mathrm{keV}$ passband; $L_{\mathrm{x}}$ refers to the distance given in Table 1 and has been corrected from the encircled PSF fraction.

even above the expectation. Wolk et al. (2005) derived a rate of 1 flare per $640 \mathrm{ks}$ from a detailed variability analysis of young solar-analogs in Orion. However, due to the observational limitations in our short snapshots we do not give a lot of weight to this difference, and, more generally, with this data set variability cannot be used to examine the origin of the X-rays.

\subsection{X-ray spectra}

For most of the X-ray sources in the sample presented here the number of counts collected is small but sufficient for a basic description of the temperature and luminosity of the emitting plasma. As shown by Feigelson et al. (2002) for data of similar quality the typical uncertainties in $k T$ for sources between 30 


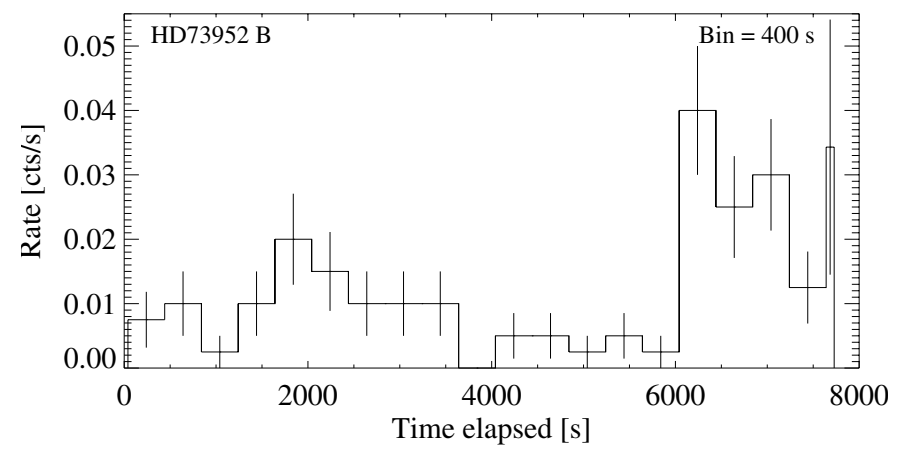

Fig. 3. Chandra ACIS lightcurve of HD 73952 B, the only significantly variable star in the sample.

and 100 counts with ACIS are about $30 \ldots 60 \%$. Thus, although limited in detailed information, the derived parameters are not meaningless. In our earlier study (SHH03) we have shown the improvement in the estimate of the spectral parameters obtained with similar quality ACIS spectra versus earlier observations of ROSAT.

For all but the faintest X-ray sources from Table 4 we extracted a spectrum, the corresponding detector response matrix that maps pulse heights into energy space, and an auxiliary response file that contains information about the effective area and detector efficiency across the chip as a function of energy. We binned each spectrum to a minimum of 5 or more counts per bin starting at $0.5 \mathrm{keV}$. As the background of ACIS is very low ( $<1$ count in the source extraction area) it can be neglected. Spectral modelling was performed in the XSPEC environment, version 11.3.0.

First we approximated each spectrum with a onetemperature (1-T) thermal model $\left(\mathrm{APEC}^{4}\right)$. Some of the spectra have a high-energy excess with respect to the 1-T model. These spectra are better described when a second component with higher temperature is added, as verified by a significant reduction of $\chi_{\text {red }}^{2}$ as well as visual inspection. Since the hot component is required only for the brightest sources, its apparent absence in the remainder of the spectra can likely be attributed to the poorer signal at high energies, rather than being a true physical difference. A direct comparison shows that for most spectra the temperature from the 1-T model agrees reasonably well with the mean temperature of the 2-T model, defined as the average of the two temperatures weighted with the emission measures. This test yields a justification for the wide-spread use of 1-T models in cases of poor statistics. However, to obtain a consistent description of all spectra, we always choose the 2 -T model as the best fit (see Table 5).

Extinction is likely to be negligible because the stars are evolved enough such that no substantial amount of circumstellar material is expected to be present. In fact, HD 129791 is the only star with measured non-vanishing optical extinction. To test the role of absorption in the X-ray spectra we include a photoabsorption term comprising the atomic cross-section and elemental abundances from Wilms et al. (2000). Two sets of spectral fits were performed, one with free column density $\left(N_{\mathrm{H}}\right)$ and one with $N_{\mathrm{H}}$ set to zero. The results show that the model without absorption is adequate, i.e. the model with free column density either yields $N_{\mathrm{H}}=0$ or the fit is poorer (based on the $\chi^{2}$ statistics and visual inspection of the residuals). An exception is HD $129791 \mathrm{~A}$. Here, a photo-absorption term is required to

4 For a description of the Astrophysical Plasma Emission Code (APEC) see Smith et al. (2001). describe the spectral shape below $1 \mathrm{keV}$ (see Fig. 4). HD $133880 \mathrm{~B}$ and HD $113703 \mathrm{~B}$ may be better described with a 3-T model - see SHH03 for a graphical representation of their $\mathrm{X}$-ray spectra - but the highest temperature does not make a large contribution to the total emission measure, such that we can safely ignore it.

The luminosities in Table 5 refer to the $0.5-8.0 \mathrm{keV}$ broadband, after correction for absorption in the case of HD $129791 \mathrm{~A}$. These luminosities have a tendency to be slightly higher than the values derived with PIMMS assuming an iso-thermal plasma of temperature $1 \mathrm{keV}$. The emission measure weighted mean temperatures extracted from the spectra are generally slightly smaller than $1 \mathrm{keV}$. But this is expected to produce an opposite trend, i.e. $L_{\mathrm{x}}$ is expected to be smaller. However, the discrepancy is only $0.1-0.2$ dex in $\log L_{\mathrm{x}}$, and may be ascribed to the use of different plasma codes: RS (Raymond \& Smith 1977) within PIMMS and APEC for the spectral modeling.

\section{The $L_{x} / L_{\text {bol }}-$ relation}

Figure 5 displays the $L_{\mathrm{x}} / L_{\text {bol }}$ ratio for all components. In the sample investigated here the companions display $\log \left(L_{\mathrm{x}} / L_{\mathrm{bol}}\right)$ values near the saturation limit. The spread is remarkably small, compared to the 2-3 dex observed typically for late-type preMS stars. Judging from the age of the primaries (on the order of $10-100 \mathrm{Myr})$, the companions are probably relatively evolved and non-accreting. The absence of accretors diminuishes the scatter of $L_{\mathrm{x}} / L_{\mathrm{bol}}$ in a sample of pre-MS stars because the latter ones have systematically lower X-ray luminosities; see e.g. Preibisch et al. (2005).

For all intermediate-mass stars of the sample that are not detected, the upper limits we derive are lower than the canonical value of $10^{-7}$ for hot stars. Although only one of the four nondetections is clearly below this value, we conjecture that none of these sources are wind-driven. The detected B/A stars show values of $\log \left(L_{\mathrm{x}} / L_{\mathrm{bol}}\right)$, which are somewhat higher than expected for "classical" wind X-ray sources. However, we caution that recent studies have demonstrated that not all hot stars fullfill the $\log \left(L_{\mathrm{x}} / L_{\mathrm{bol}}\right)=10^{-7}$ relation (Stelzer et al. 2005b).

In terms of absolute X-ray luminosity the detected intermediate-mass stars and the companions are indistinguishable, with the majority showing $L_{\mathrm{x}} \sim 10^{29 . .30} \mathrm{erg} / \mathrm{s}$. This is indicated by the shaded area in Fig. 5. Assuming that the X-ray emission from the B/A stars is produced by as yet unknown close cool magnetically active companions which emit at typical levels of $\log \left(L_{\mathrm{x}} / L_{\text {bol }}\right) \sim-3.5$, we find bolometric luminosities between $\log \left(L_{\mathrm{bol}} / L_{\odot}\right) \sim 0.0 \ldots-1.5$ for such objects. As can be seen from Table 3 this is the range of $L_{\mathrm{bol}}$ for the companions that have already been identified and spatially resolved.

\section{Discussion}

In this paper we have presented the Chandra observations of 6 late-B stars on the MS. The sample complements a previous survey of 5 B-type stars presented by SHH03. The targets are characterized by (1) being known to emit X-rays from ROSAT, and (2) having close companions that could not be spatially separated in previous X-ray images, leaving doubt on the origin of their ROSAT detection. We find that the B-type primary is detected in 7 out of 11 cases, after resolving it from all known visual companions with Chandra. A trend of the early-type stars to split in two groups was pointed out by Daniel et al. (2002) for the Pleiades: Apparent X-ray emitters on the one side, and on the 
Table 5. Spectral parameters of X-ray sources associated with B/A stars and/or their known close companions. The absorption is negligible, except for HD $129791 \mathrm{~A}$. The higher temperature of HD $1685 \mathrm{~B}$ is unconstrained and the $90 \%$ confidence lower limit is given.

\begin{tabular}{lcrrrrrrr}
\hline \hline HD & Opt/IR & $\chi_{\text {red }}^{2}$ (d.o.f.) & $\begin{array}{r}\log N_{\mathrm{H}} \\
{\left[\mathrm{cm}^{-2}\right]}\end{array}$ & $\begin{array}{r}k T_{1} \\
{[\mathrm{keV}]}\end{array}$ & $\begin{array}{r}k T_{2} \\
{[\mathrm{keV}]}\end{array}$ & $\begin{array}{r}\log E M_{1} \\
{\left[\mathrm{~cm}^{-3}\right]}\end{array}$ & $\begin{array}{r}\log E M_{2} \\
{\left[\mathrm{~cm}^{-3}\right]}\end{array}$ & $\begin{array}{r}\log L_{\mathrm{x}}{ }^{*} \\
{[\mathrm{erg} / \mathrm{s}]}\end{array}$ \\
\hline HD 32964 & B & $0.87(5)$ & - & 0.54 & 1.62 & 52.24 & 52.16 & 29.41 \\
HD 73952 & B & $1.13(12)$ & - & 0.68 & 1.26 & 52.19 & 51.97 & 29.33 \\
HD 73952 & A & $0.87(4)$ & - & 0.32 & 1.04 & 51.98 & 51.75 & 29.04 \\
HD 110073 & B & $0.32(4)$ & - & 0.62 & 1.03 & 52.36 & 51.79 & 29.35 \\
HD 134946 & A & $0.80(10)$ & - & 0.69 & 1.30 & 52.68 & 52.63 & 29.91 \\
HD 129791 & A & $0.89(47)$ & 21.23 & 0.64 & 1.49 & 53.38 & 53.02 & 30.36 \\
HD 129791 & L & $0.63(13)$ & - & 0.51 & 1.15 & 52.42 & 52.19 & 29.53 \\
\hline HD 113703 & L & $1.15(73)$ & - & 0.80 & 4.32 & 53.15 & 52.87 & 30.30 \\
HD 113703 & B & $1.83(40)$ & - & 0.34 & 0.99 & 52.83 & 52.39 & 29.77 \\
HD 1685 & B & $0.95(8)$ & - & 0.70 & $>0.88$ & 51.89 & 52.01 & 29.40 \\
HD 1685 & A & $1.22(3)$ & - & 0.25 & 1.06 & 52.34 & 51.69 & 29.12 \\
HD 123445 & B/C & $0.79(6)$ & - & 0.43 & 0.98 & 52.20 & 52.75 & 29.94 \\
HD 133880 & B & $1.95(13)$ & - & 0.20 & 1.00 & 52.79 & 52.72 & 29.94 \\
HD 169978 & A & $0.87(7)$ & - & 0.38 & 1.29 & 53.01 & 52.61 & 29.95 \\
\hline
\end{tabular}

* In the $0.5-8 \mathrm{keV}$ passband; $L_{\mathrm{x}}$ refers to the distance given in Table 1 .

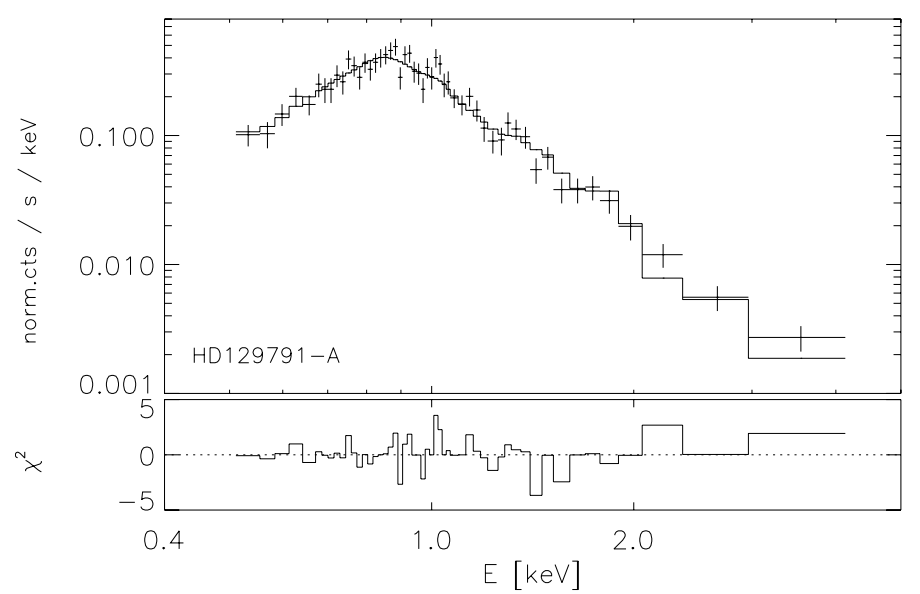

Fig. 4. X-ray spectrum of HD 129791 A: data, best fit model, and residuals. The 2-T fit requires an absorption term with $N_{\mathrm{H}}=1.7 \times 10^{21} \mathrm{~cm}^{-2}$.

other side X-ray quiet stars with upper limits by 1-2 orders of magnitudes lower than the $L_{\mathrm{x}} / L_{\text {bol }}$ values of the detected B-type stars. Our observations are consistent with this general picture, although the two populations are not clearly separated. The two possible interpretations for the observed behavior are (i) X-ray emission is intrinsic to some B-type stars, or (ii) the detected late-B type stars have further (as yet undiscovered) low-mass companions.

The detection fraction of $>60 \%$ for the primary stars is surprisingly high. We compare these numbers with the sample of RASS detected late-B type stars. The catalog by BSC96 counts 121 X-ray detected stars with spectral types between B5...B9 versus 973 non-detections in the same spectral type range (12\% detection rate). A systematic search of the literature revealed that for 76 among the 121 RASS detections, closeby fainter companions are known that are confused with the B-type star in the ROSAT error box. The Chandra targets are drawn from this sample. Therefore, it seems justified to assume that our B star detection rate of $>60 \%$ is applicable to all known visual late$\mathrm{B}$ type binaries. If the residual $\mathrm{X}$-ray emission at the primary

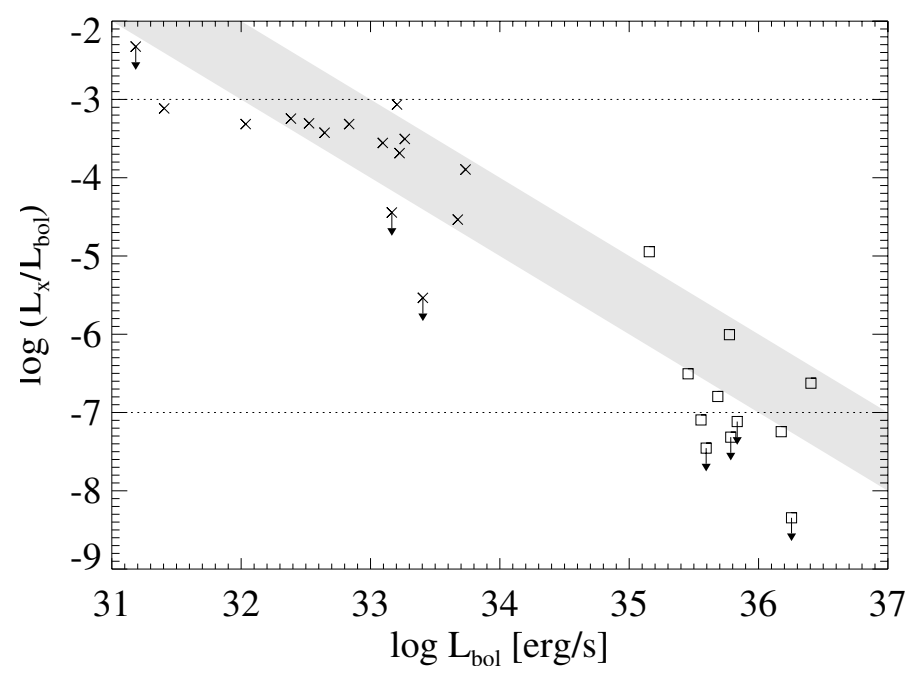

Fig. 5. Ratio between X-ray and bolometric luminosity for A- and B-type stars (squares) and their companions (X-points). The shaded area indicates the position of objects in the range $L_{\mathrm{x}}=10^{29 \ldots 30} \mathrm{erg} / \mathrm{s}$. Dotted lines indicate the canonical value for $L_{\mathrm{x}} / L_{\mathrm{bol}}$ of late-type stars $\left(10^{-3}\right)$ and of hot stars $\left(10^{-7}\right)$. For the unresolved pair of companions HD 123445 B and C it was assumed that the observed X-ray luminosity is distributed equally over both stars.

position is attributed to unknown companions, this implies that about $(76 / 121) \times(7 / 11) \sim 40 \%$ of X-ray detected B-type stars are high multiplicity systems. This seems at first hand unlikely. However, a speckle survey has revealed a fraction of $\sim 50 \%$ of high-order multiples among known spectroscopic binaries of HgMn type (Isobe 1991). Furthermore, the near-IR studies by Hubrig et al. (2005) have indicated the discovery of third components in many SB systems among chemically peculiar (CP) stars.

In the Chandra sample three stars are known to have CP character: HD 32964 and HD 110073 are HgMn stars, and both are spectroscopic binaries, i.e. taking into account their visual companions both systems are probably at least triples. 
Interestingly, HD 32964 is composed of two nearly equal mass B-type stars, that apparently both are X-ray dark, because the only X-ray source is associated with the visual companion. HD 110073, is detected with Chandra, but it might have a magnetic field (see below), suggestive of X-ray production on the intermediate-mass star itself. The third CP star is HD 133880, an Ap star which has no known spectroscopic companion, and it is also not detected in X-rays.

As an alternative to the companion hypothesis, the high $\mathrm{X}$-ray detection fraction of the primaries may indicate that - contrary to common belief - at least some of them are capable of producing high-energy emission. Intermediate-mass stars possess neither strong winds nor a solar-type dynamo, but they may represent a hybrid case. A magnetically confined wind has been proposed by Babel \& Montmerle (1997) to explain the unusually strong X-ray luminosity from the magnetic Ap star IQ Aur. In contrast to the classical wind-shock picture, this model can also produce relatively hard X-ray emission, with temperatures in excess of $10^{7} \mathrm{~K}$ produced in shocks that form upon collision of the magnetically channeled winds from the two stellar hemispheres. The high observed X-ray temperatures of the Chandra sources identified with the primaries $(\sim 10 \mathrm{MK})$ therefore do not exclude that the emission comes from the B stars. But, obviously, the general applicability of this scenario to A- and B-type stars depends on the presence of magnetic fields. Their detection is difficult in intermediate-mass stars because they have fewer absorption lines and faster rotation rates than lower-mass stars. Bychkov et al. (2003) have compiled a list of field measurements for $\sim 600 \mathrm{MS}$ and giant stars. This catalog comprises 22 of the 121 X-ray emitting late-B stars from BSC96, of which 8 are apparently single stars.

Two stars of the Chandra sample are listed by Bychkov et al. (2003), but they behave contrary to the expectation: The field measurement for HD 110073 is barely significant. This star is detected with Chandra, but it is a spectroscopic binary. HD 133880 is an Ap star with a strong, probably non-dipolar field $(2-4 \mathrm{kG}$; Landstreet 1990; Bychkov et al. 2003), but in SHH03 we have shown that the X-ray emission can be attributed to the companion. Therefore, the presence of magnetic fields does not necessarily imply a relation to X-ray emission.

The short exposure times of the Chandra observations do not allow us to examine X-ray properties of the detected sources in detail. Therefore, it is difficult to tell if the X-ray emission from the position of the primaries differs qualitatively from the X-rays produced by the resolved companions. Comparison of the X-ray luminosities and temperatures for the primaries on the one hand and the companions on the other hand does not indicate any systematic trend (cf. Table 5 and Fig. 5). This is consistent with the same emission mechanism working in both groups. Collectively, the X-ray luminosities and temperatures of the objects studied in this paper are much lower than those of low-mass pre-MS stars in star forming regions (e.g. Preibisch \& Zinnecker 2002; Getman 2002; Feigelson et al. 2002). When choosing energies of $0.5-2.0 \mathrm{keV}$ for the soft band and $2.0-8.0 \mathrm{keV}$ for the hard band, as common for pre-MS objects, all our targets show very negative hardness ratios $(H R=-0.8$ and below $)$, indicating that no significant emission is present at high energies. Thus, both spectral hardness and X-ray luminosity put our targets between the Pleiades and the bulk of pre-MS stars. If this is to be interpreted as an evolutionary effect the low-mass stars in the sample presented here should be in their final approach to the MS, in agreement with their evolutionary state derived from the IR magnitudes and colors.
The fact that nearly all known resolved companion candidates are shown to be X-ray sources, and the similarity of their $\mathrm{X}$-ray properties to those of the B-type primaries, motivates us to speculate about the potential of such objects in eventually explaining the observed X-ray emission from all or most MS B-type stars. In this vein, X-ray detections could be used as a tool to discover faint companions to B-type stars. The most likely exceptions are the Ap/Bp stars. Checking whether they form a class of intrinsic X-ray emitters must include a systematic study of their multiplicity. In future work we will continue to examine the multiplicity of the apparently X-ray active B-type stars through IR imaging (see Stelzer et al. 2005a; Huélamo et al. 2005, for preliminary results) and IR/optical spectroscopy. Further high resolution X-ray observations are also desirable. Combining these techniques on a large sample is essential to solve the longstanding mystery of the X-rays from intermediatemass stars.

Acknowledgements. Very special thanks to Kevin Briggs for original thoughts that we tried to incorporate in this work. We would like to thank the anonymous referee for useful comments. This publication makes use of data products from the Two Micron All Sky Survey, which is a joint project of the University of Massachusetts and the Infrared Processing and Analysis Center/California Institute of Technology, funded by the National Aeronautics and Space Administration and the National Science Foundation. This research has made use of the SIMBAD database, operated at CDS, Strasbourg, France, and the Hipparcos catalogue accessed through the VizieR data base.

\section{References}

Abt, H. A., Levato, H., \& Grosso, M. 2002, ApJ, 573, 359 Andersen, J., \& Nordstrom, B. 1983, A\&AS, 52, 471

Babel, J., \& Montmerle, T. 1997, A\&A, 323, 121

Babel, J. 1996, A\&A, 309, 867

Baraffe, I., Chabrier, G., Allard, F., \& Hauschildt, P. H. 1998, A\&A, 337, 403 Berghöfer, T. W., \& Schmitt, J. H. M. M. 1994, A\&A, 292, L5

Berghöfer, T. W., Schmitt, J. H. M. M., \& Cassinelli, J. P. 1996, A\&AS, 118, 481 (BSC96) Bychkov, V. D., Bychkova, L. V., \& Madej, J. 2003, A\&A, 407, 631

Cash, W., \& Snow, T. P. 1982, ApJ, 263, L59

Catanzaro, G., \& Leto, P. 2004, A\&A, 416, 661

Caillault, J.-P., \& Zoonematkermani, S. 1989, ApJ, 338, L57

Close, L. M., Richer, H. B., \& Crabtree, D. R. 1990, AJ, 100, 1968

Cutri, R. M., Skrutskie, M. F., Van Dyk, S., et al. 2003, The 2MASS All-Sky Catalog of Point Sources, University of Massachusetts and Infrared Processing and Analysis Center (IPAC/California Institute of Technology)

Daniel, K. J., Linksy, J. L., \& Gagné, M. 2002, ApJ, 578, 486

Dodd, R. J. 2004, MNRAS, 355, 959

de Geus, E. J., de Zeeuw, P. T., \& Lub, J. 1989, A\&A, 216, 44

de Zeeuw, P. T., Hoogerwerf, R., de Bruijne, J. H. J., Brown, A. G. A., \& Blaauw, A. 1999, AJ, 117, 354

Duquennoy, A., \& Mayor, M. 1991, A\&A, 248, 485

Eggen, O. J. 1963, AJ, 68, 483

Feigelson, E. D., Broos, P., Gaffney, J. A., et al. 2002, ApJ, 574, 258

Freeman, P. E., Kashyap, V., Rosner, R., \& Lamb, D. Q. 2002, ApJS, 138, 185

Frost, E. B., \& Struve, O. 1924, ApJ, 60, 313

Gahm, G. F., Ahlin, P., \& Lindroos, K. P. 1983, A\&AS, 51, 143

Gehrels, N. 1986, ApJ, 303, 336

Gerbaldi, M., Faraggiana, R., \& Balin, N. 2001, A\&A, 379, 162

Getman, K. V., Feigelson, E. D., Townsley, L., et al. 2002, ApJ, 575, 354

Hogg, A. R. 1960, PASP, 72, 85

Hubrig, S., Le Mignant, D., North, P., \& Krautter, J. 2001, A\&A, 372, 152

Hubrig, S., Ageorges, N., \& Schöller, M. 2005, in Proc. of Multiple Stars across the H-R Diagram, ESO Astrophysics Symposia, in press [arXiv: astro-ph/0510302]

Huélamo, N., Neuhäuser, R., Stelzer, B., Supper, R., \& Zinnecker, H. 2000, A\&A, 359, 227

Huélamo, N., Brandner, W., Brown, A. G. A., Neuhäuser, R., \& Zinnecker, H. 2001, A\&A, 373,657

Huélamo, N., Chauvin, G., Foellmi, C., et al. 2005, in Proc. of Multiple Stars across the HR Diagram, Jul. 12-15, 2005, Garching, Germany, ESO Astrophysics Symposia, in press

Hünsch, M. 2001, in Proc. of the 11th Workshop on Cool Stars, Stellar Systems and the Sun, ed. R. J. García Lopez, R. Rebolo, \& M. R. Zapatero Osorio, ASP Conf. Ser., 223, 967 Hünsch, M., Schmitt, J. H. M. M., \& Voges, W. 1998, A\&AS, 132, 155

Isobe, S. 1991, PASAu, 9, 270

Kenyon, S., \& Hartmann, L. 1995, ApJS, 101, 117

Kouwenhoven, M. B. N., Brown, A. G. A., Zinnecker, H., Kaper, L., \& Portegies Zwart, S. F. 2005, A\&A, 430, 137

Kraft, R. P., Burrows, D. N., \& Nousek, J. A. 1991, ApJ, 374, 344 
Landstreet, J. D. 1990, ApJ, 352, L5

Lindroos, K. P. 1983, A\&AS, 51, 161

Lindroos, K. P. 1985, A\&AS, 60, 183

Lindroos, K. P. 1986, A\&A, 156, 223

Lucy, L. B., \& White, R. L. 1980, ApJ, 241, 300

Owocki, S. P., \& Cohen, D. H. 1999, ApJ, 520, 833

Palla, F., \& Stahler, S. W. 1999, ApJ, 525, 772

Pallavicini, R., Pasquini, L., \& Randich, S. 1992, A\&A, 261, 245

Parker, E. N. 1955, ApJ, 122, 293

Parker, E. N. 1993, ApJ, 408, 707

Patten, B. M., \& Simon, Th. 1993, ApJ, 415, L123

Preibisch, T., \& Zinnecker, H. 2002, AJ, 123, 1613

Preibisch, T., Kim, Y.-C., Favata, F., et al. 2005, ApJS, 160, 401

Raymond, J. C., \& Smith, B. W. 1977, ApJS, 35, 419

Renson, P., Gerbaldi, M., \& Catalano, F. A. 1991, A\&AS, 89, 429

Royer, F., Gerbaldi, M., Faraggiana, R., \& Gomez, A. E. 2002, A\&A, 381, 105

Rüdiger, G., \& Brandenburg, A. 1995, A\&A, 296, 557

Ryter, Ch. E. 1996, Ap\&SS, 236, 285

Schmitt, J. H. M. M., \& Liefke, C. 2004, A\&A, 417, 651

Schmitt, J. H. M. M., Golub, L., Harnden, F. R. Jr., et al. 1985, ApJ, 290, 307
Schmitt, J. H. M. M., Zinnecker, H., Cruddace, R., \& Harnden, F. R. Jr. 1993, ApJ, 402, L13 Schneider, H. 1981, A\&A, 44, 137

Shatsky, N., \& Tokovinin, A. 2002, A\&A, 382, 92

Smith, R. K., Brickhouse, N. S., Liedahl, D. A., \& Raymond, J. C. 2001, ApJ, 556, L91

Stelzer, B., \& Neuhäuser, R. 2001, A\&A, 377, 538

Stelzer, B., Huélamo, N., Hubrig, S., Zinnecker, H., \& Micela, G. 2003, A\&A, 407, 1067 (SHH03)

Stelzer, B., Micela, G., \& Neuhäuser, R. 2004, A\&A, 423, 1029

Stelzer, B., Micela, G., Huélamo, N., \& Hünsch, M. 2005a, in Proc. of Multiple Stars across the HR Diagram, Jul. 12-15 2005, Garching, Germany, ESO Astrophysics Symposia, in press

Stelzer, B., Flaccomio, E., Montmerle, T., et al. 2005b, ApJS, 160, 557

Turon, C., Egret, D., Gomez, A., et al. 1993, Hipparcos Input Catalogue, Version 2, Bull. Inf. CDS 43, 5

Uesugi, A., \& Fukuda, I. 1970, Contr. Astroph. Kwasan Obs. Univ. Kyoto, 189

Wielen, R., Dettbarn, Ch., Lenhardt, H., et al. 2000, Veröffentlichungen Astronomisches Rechen-Institut Heidelberg, Verlag G. Braun, Karlsruhe, No. 37, 1

Wilms, J., Allen, A., \& McCray, R. 2000, ApJ, 542, 914

Wolk, S. J., Harnden, F. R. Jr., Flaccomio, E., et al. 2005, ApJS, 160, 423

Yushchenko, A. V., Gopka, V. F., Khokhlova, V. L., \& Tomkin, J. 2001, IBVS, 5213, 1 
B. Stelzer et al.: X-ray emission from main-sequence B-stars, Online Material $p 1$

\section{Online Material}




\section{Appendix A: Individual targets}

\section{A.1. HD 32964}

HD 32964 (=66 Eri) is a CP star of HgMn type (Renson 1991), and a spectroscopic binary (Frost \& Struve 1924) composed of two nearly equal mass stars of $M \sin ^{3} i \sim 2.4 \mathrm{M}_{\odot}$ in a 5.2-day orbit (Yushchenko et al. 2001; Catanzaro \& Leto 2004).

HD 32964 is also a Lindroos system with a secondary at a separation of $\sim 53^{\prime \prime}$. Lindroos (1985) noted that common proper motion is observed, but Eggen (1963) classified the K5 V secondary as optical, and based on this classification it was further on discarded from the Lindroos sample. Consequently, this star was not included in the X-ray study of Huélamo et al. (2000). In the RASS an X-ray source was detected with $\log L_{\mathrm{x}}=29.8 \mathrm{erg} / \mathrm{s}$ (Berghöfer et al. 1996). HD 32964 was also part of the ROSAT HRI sample examined by Berghöfer \& Schmitt (1994). While the Lindroos secondary remained undetected consistent with its classification as non-physical companion, the primary was detected at a level of $L_{\mathrm{x}}=7 \times 10^{29} \mathrm{erg} / \mathrm{s}$.

After the ROSAT study another, presumably late-type, companion to HD 32964 was discovered by Hubrig et al. (2001) with AO observations. Near-IR photometry puts the object near the zero-age MS in the CMD (Fig. 2), in agreement with the relatively old age ( 200 Myr) derived for the primary by Hubrig et al. (2001). With a separation of only $\sim 1.6^{\prime \prime}$ this object was not resolvable from the primary with the ROSAT HRI. The only X-ray source detected with Chandra in the vicinity of HD 32964 is clearly identified with this companion. The upper limit for the B-type primary is $L_{\mathrm{x}}<1.6 \times 10^{28} \mathrm{erg} / \mathrm{s}$. The Lindroos secondary remains undetected at an upper limit of $L_{\mathrm{x}}<8 \times 10^{27} \mathrm{erg} / \mathrm{s}$, further evidence that it is not a young late-type star, despite it being compatible with the pre-MS tracks.

\section{A.2. $H D 73952$}

HD 73952 is a member of the 30-50 Myr-old open cluster IC 2391 (Hogg 1960; Dodd 2004). Patten \& Simon (1993) reported the X-ray detection of HD 73952 in a ROSAT pointed observation, with $L_{\mathrm{x}}$ four times overluminous according to the canonical relation of $L_{\mathrm{x}} / L_{\mathrm{bol}}=10^{-7}$ for early-type stars. Berghöfer et al. (1996) list HD 73952 with an X-ray luminosity of $\log L_{\mathrm{x}}=29.7 \mathrm{erg} / \mathrm{s}$.

HD 73952 is not a RV variable according to Andersen et al. (1983). Recent AO observations revealed a faint IR object at 1.2" from the B-type star (Hubrig et al. 2001). Its position in the $J H K$ CMD suggests that it is a very young star of very low mass.

The Chandra image shows two X-ray sources coincident with either of the two components in the HD 73952 system. The brighter source is associated with the secondary, and displays a typical "late-type" $L_{\mathrm{x}} / L_{\text {bol }}$ ratio. For the B-star we measure $\log \left(L_{\mathrm{x}} / L_{\mathrm{bol}}\right)=-6.7$, clearly - and not surprisingly - lower than indicated by ROSAT.

\section{A.3. $H D 110073$}

HD 110073 is a CP star of the HgMn class (Renson 1991). An averaged quadratic magnetic field of $145 \pm 158 \mathrm{G}$ was reported (Bychkov et al. 2003) combining earlier measurements from the literature. As can be seen from the large $1 \sigma$ uncertainty the detection of the magnetic field is highly uncertain. A faint IR object at a separation of $1.2^{\prime \prime}$ seems to be a comparatively massive $>1 M_{\odot}$ star on the pre-MS (Hubrig et al. 2001). Furthermore,
HD 110073 is a single-lined spectroscopic binary (Schneider 1981).

Two close X-ray sources are detected with Chandra, spatially coincident with the optical/IR position of HD $110073 \mathrm{~A}$ and the companion discovered in the AO observations. The presence of the spectroscopic companion casts some doubt on the origin of the X-rays from the position of the primary, while the possible detection of a magnetic field makes it an interesting candidate for being an intrinsic B-star X-ray emitter.

\section{A.4. $H D 134837$}

HD 134837 is a member of the Upper Centaurus Lupus (UCL) association (de Zeeuw et al. 1999). It was not known to be a multiple prior to the AO survey by Hubrig et al. (2001), which revealed an IR object at $4.7^{\prime \prime}$ from the B-star. The same object was recovered in a recent $\mathrm{AO}$ study of the Sco OB association by Kouwenhoven et al. (2005).

In the Chandra image both the primary and the IR object are detected as faint sources.

\section{A.5. $H D 134946$}

HD 134946 is a poorly studied late-B type star for which Hubrig et al. (2001) identified a possible companion at 8.2" separation. In our Chandra snapshot both objects are detected, although the companion only marginally ( 5 photons corresponding to $\left.\log L_{\mathrm{x}}=28.3 \mathrm{erg} / \mathrm{s}\right)$. The primary is the brighter X-ray source with an $L_{\mathrm{x}} / L_{\mathrm{bol}}$ ratio of $\sim-6$.

\section{A.6. $H D 129791$}

HD 129791 is a member of the UCL association, and a Lindroos system (Lindroos 1983). According to Gahm et al. (1983) the secondary has spectral type K5 V, and in a spectroscopic study by Pallavicini et al. (1992) it showed signs of youth (Ca II and $\mathrm{H} \alpha$ emission, strong Lithium feature). Its separation from the B9 V primary is $\sim 35^{\prime \prime}$. Both the primary and the secondary of this system were detected with ROSAT (Huélamo et al. 2000). This led Huélamo et al. (2001) to search for a further, closer companion to HD 129791 A with AO imaging, but the result was negative. Another companion search using AO by Kouwenhoven et al. (2005) was also negative.

The $\sim 7 \mathrm{ks}$ long Chandra exposure confirms the detection of both components, but no other X-ray source near the primary is detected, consistent with the absence of further companions in the IR. The primary of HD 129791 is the X-ray brightest of the B-star sample, both in absolute numbers and in terms of the fractional X-ray luminosity $\left(\log \left(L_{\mathrm{x}} / L_{\mathrm{bol}}\right) \sim-5\right)$. 